\title{
Role of Repeat Resection in Non-Muscle-Invasive Bladder Cancer
}

\author{
Harry W. Herr, MD
}

\begin{abstract}
Repeat transurethral resection (TUR) is indicated for high-grade non-muscle-invasive bladder tumors. Repeat TUR is a diagnostic, therapeutic, prognostic, and predictive procedure. Repeat TUR achieves optimal local control by removing residual tumors after initial TUR, improves staging accuracy, provides additional histologic material favoring accurate diagnosis, allocates appropriate therapy with improved outcomes, facilitates response to intravesical therapy, and provides important prognostic information. (J Natl Compr Canc Netw 2015;13:1041-1046)
\end{abstract}

Non-muscle-invasive bladder cancers (NMIBCs) account for $75 \%$ of new cases. These include non-minimally invasive (Ta) and minimally invasive (T1) papillary tumors and flat carcinoma in situ (CIS). Papillary bladder tumors are classified as low-grade (LG) or highgrade (HG). CIS is a HG lesion usually associated with papillary tumors. NMIBCs frequently recur, usually within 2 to 3 years, and $5 \%$ to $30 \%$ progress to muscleinvasion. Tumor recurrence and progression increase burden and cost of therapy, affect quality of life, and reduce survival.

Transurethral resection (TUR) is the essential surgical procedure used to diagnose, stage, and initially treat primary and recurrent NMIBC. TUR aims to provide adequate tissue to evaluate histologic type and grade of bladder tumors; determine presence and depth of tumor invasion; and remove visible and microscopic

From Memorial Sloan Kettering Cancer Center, New York, New York.

Submitted September 23, 2014; accepted for publication January 1, 2015.

The author has disclosed that he has no financial interests, arrangements, affiliations, or commercial interests with the manufacturers of any products discussed in this article or their competitors.

Correspondence: Harry W. Herr, MD, Memorial Sloan Kettering Cancer Center, 353 East 68th Street, New York, NY 10065.

E-mail: herrh@mskcc.org superficial and invasive tumors. The findings direct further therapy, dictate follow-up schedules, and indicate prognosis. ${ }^{1}$

Although TUR is a frequently performed operation familiar to urologists, it is subject to unquantifiable surgeon, tumor, and pathologic-related variables; as a result, its diagnostic and therapeutic purposes are not always achieved. NCCN Clinical Practice Guidelines in Oncology (NCCN Guidelines) for Bladder Cancer recommend a second or repeat TUR ${ }^{2}$ (within 6 weeks) in the following circumstances:

- Incomplete initial TUR,

- No muscle in original specimen for HG tumors,

- Large or multifocal tumors, or

- Any T1 tumor.

\section{Rationale for Repeat TUR}

Successful management of NMIBC is driven by 2 overarching principles, one dependent on the other. First, all visible tumors, especially invasive T1 lesions, should be completely resected. Second, intravesical therapy is most effective after complete tumor resections. Despite these caveats, residual tumor is frequently found on contemporary repeat TUR, and persistent tumors are commonly found at cystoscopy 3 months after diagnosis, suggesting incomplete initial resection. A third variable affecting outcomes of NMIBC is pathologic evaluation of tumor specimens. Although pathologists may differ in their interpretation, they can only assess what they are given by the urologist. As an example, one study found that muscularis propria was missing in up to $51 \%$ of TUR specimens. ${ }^{3}$ Furthermore, tumors are often submitted in multiple pieces, from which pathologists are asked to reconstruct the whole tumor type, configuration, and extent. The more specimens submitted from tumor sites and margins, the more like- 
Herr

ly pathologists are able to correctly define tumor boundaries. Although the uncertainties inherent in the stochastic nature of TUR cannot be eliminated entirely, they can be substantially reduced by a timely repeat resection.

Does quality of initial TUR or expertise of the urologist matter? Even experienced urologists have a high percentage of persistent carcinoma after performing the initial TUR. Of 214 patients undergoing restaging TUR for Ta or T1 tumors, carcinoma was present in 37\% treated by senior urologists and $26 \%$ treated by urologists in training. ${ }^{4}$ We performed both initial and repeat TUR in 71 patients with T1 disease and found that $17 \%$ had persistent tumor invading the lamina propria. ${ }^{5}$ Thus, repeat TUR is indicated in all high-risk patients being considered for conservative management to allow better risk assessment.

The question is why is there a high rate of persistent tumor after initial TUR for NMIBC, and why can't even experienced urologists do it right the first time? The answer lies in the technical difficulties of the procedure itself and in the nature of bladder tumors. Visibly complete TUR of multiple papillary lesions, especially T1 tumors associated with CIS, is often difficult the first time. Tumors may be overlooked if extensive, or may involve sometimes difficult-to-reach regions of the bladder, such as the dome, anterior wall, bladder neck, or urethra. Tumor spread at the margins or invading lamina propria is not always seen at cystoscopy and is often more extensive than surface appearance of the tumor suggests. As the resection proceeds, vision often becomes obscured, owing to mucosal edema, bladder spasms, and bleeding, making it increasingly difficult to differentiate benign from tumor-bearing mucosa, and to obtain clear negative surgical margins.
TUR is a technically difficult procedure that, combined with uncertainty of tumor growth, conspires to limit the urologist's ability to always perform a reliably complete TUR. Although we can teach ourselves and others to perform high-quality TURs, ${ }^{6}$ that alone will not completely negate the value or need for a contemporary repeat TUR for most NMIBCs. ${ }^{1}$ Perhaps new optical enhancement methods under investigation, such as fluorescence cystoscopy and narrow band imaging, or other novel technologies will help improve the performance of the initial TUR ${ }^{7}$; however, they will not solve the problem of understaging.

Repeat resection is a diagnostic, prognostic, therapeutic, and predictive procedure. Robust evidence supports each reason for repeat TUR of NMIBC.

\section{Repeat TUR Improves Diagnosis and Staging}

Repeat TUR is diagnostic because it detects disease missed on the initial resection. For example, we found residual tumors in $74 \%$ of 1312 patients who underwent second TUR. ${ }^{8}$ In patients with highgrade lesions, half of the Ta tumors had residual disease and $15 \%$ were upstaged; for T1 tumors, $48 \%$ had persistent NMIBC and 30\% were upstaged to muscle invasion (Table 1). Restaging TUR was not helpful for LG papillary tumors. Multiple, HG Ta or T1 tumors are at greater risk for incomplete resection. The second TUR staged papillary tumors more accurately and provided better local treatment of the primary lesions.

A recent meta-analysis in 2262 cases from the world literature reported residual disease on repeat TUR in $19 \%$ to $56 \%$ of Ta and $15 \%$ to $55 \%$ of T1 tumors, with tumor at the same site in $44 \%$ to $86 \%$ and at different locations in $14 \%$ to $56 \% .{ }^{9}$ Upstaging occurred in $14 \%$ of Ta and $24 \%$ of T1 tumors. Each series found repeat TUR increased tumor detection

\begin{tabular}{|llccccr|}
\hline \multirow{2}{*}{ Table 1 Restaging Ta and T1 Bladder Tumors } \\
\cline { 3 - 7 } Tumor Type & N & T0 & Ta-LG & Ta-HG/CIS & T1 & T2 \\
\hline Ta-LG & 215 & 49 & 46 & 5 & 0 & 0 \\
Ta-HG & 396 & 35 & 0 & 50 & 10 & 5 \\
T1 & 701 & 22 & 0 & 23 & 25 & 30 \\
Muscle & 421 & 25 & 0 & 31 & 29 & 15 \\
No muscle & 280 & 20 & 0 & 15 & 20 & 45 \\
\hline
\end{tabular}

Abbreviations: CIS, carcinoma in situ; HG, high-grade papillary tumors; LG, low-grade papillary tumors; TUR, transurethral resection. $62 \%$ had associated CIS. 

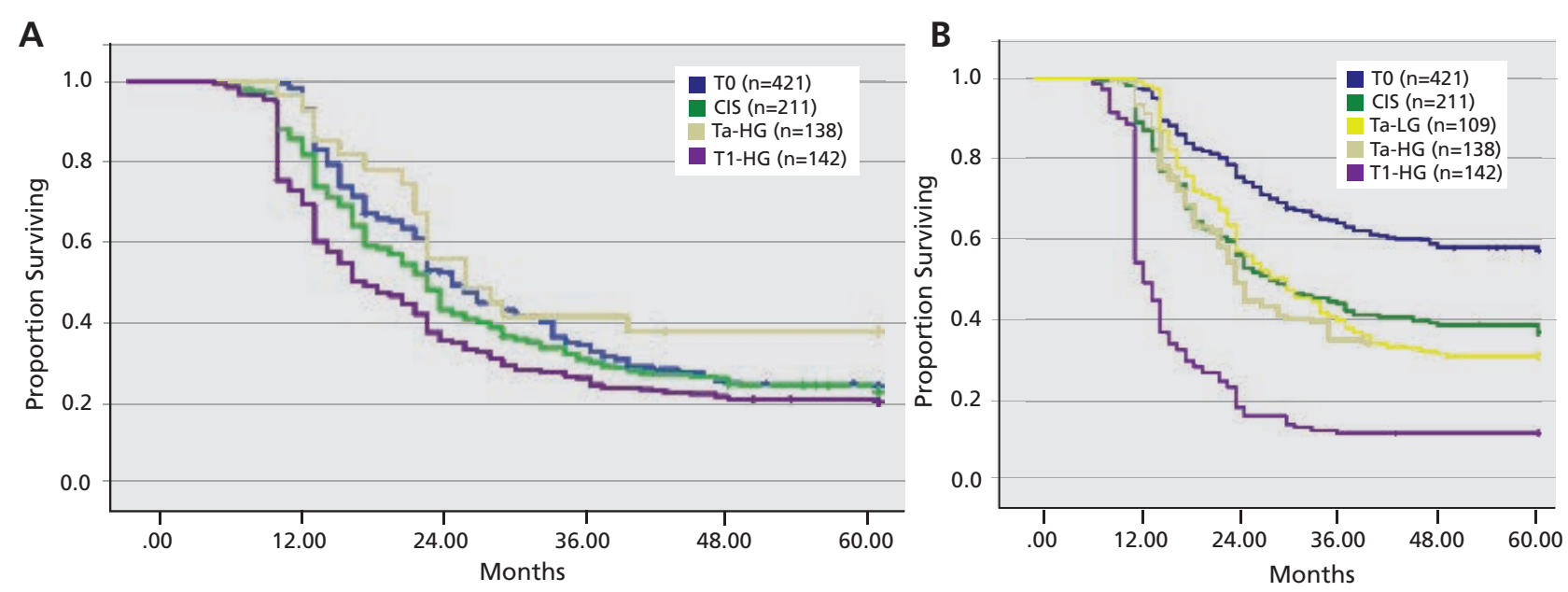

Figure 1 Recurrence-free survival of Ta-LG, Ta-HG, T1-LG, and T1-HG tumors by (A) initial TUR pathology versus (B) repeat TUR pathology. Abbreviations: CIS, carcinoma in situ; HG, high grade; LG, low grade; TUR, transurethral resection.

and improved staging accuracy. Incomplete resection is responsible for most early recurrences, providing a compelling argument for a repeat TUR.

\section{Repeat TUR Better Defines Prognosis}

Repeat TUR is prognostic because outcome is better defined. Figure 1 shows recurrence-free survival of 1021 patients with NMIBC classified as Ta-LG, TaHG, T1-LG, or T1-HG who were followed 5 years after TUR and bacillus Calmette-Guérin (BCG) therapy, stratified by initial TUR pathologic results (Figure 1A) versus pathology based on repeat TUR (Figure 1B). Half of the patients in each group experienced recurrence, and recurrence-free survival times did not differ $(P=.08)$ among the groups identified by initial TUR specimen. However, repeat TUR pathologic findings identified patients with fewer (TO) or greater (T1) recurrences. Such information can be used to modify treatments and follow-up regimens. ${ }^{8}$

Figure 2 shows progression-free survival based on initial (Figure 2A) or repeat TUR pathologic findings (Figure 2B). No difference was seen in progression between HG Ta and T1 tumors on initial TUR pathologic results; however, repeat TUR pathologic evaluation showed that $78 \%$ of patients having residual T1 disease experienced progression, including many whose disease had responded initially to BCG therapy, and a third died. Only 9\% of patients with no or less than T1 tumor on repeat TUR experienced progression, suggesting that such patients may be treated successfully with bladder-sparing intravesical therapy. Another study confirms these findings in patients who had no tumor on repeat resection had
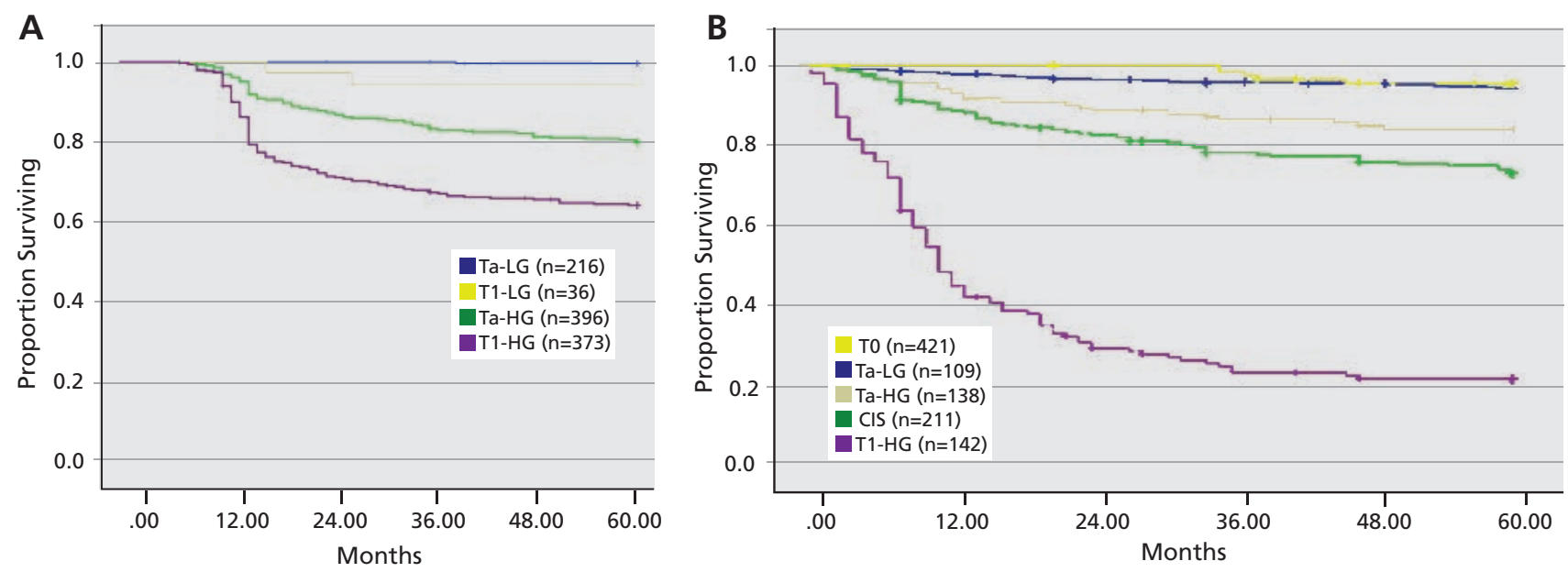

Figure 2 Progression-free survival after (A) initial TUR pathology versus (B) repeat TUR pathology. Abbreviations: CIS, carcinoma in situ; HG, high grade; LG, low grade; TUR, transurethral resection. 
Herr
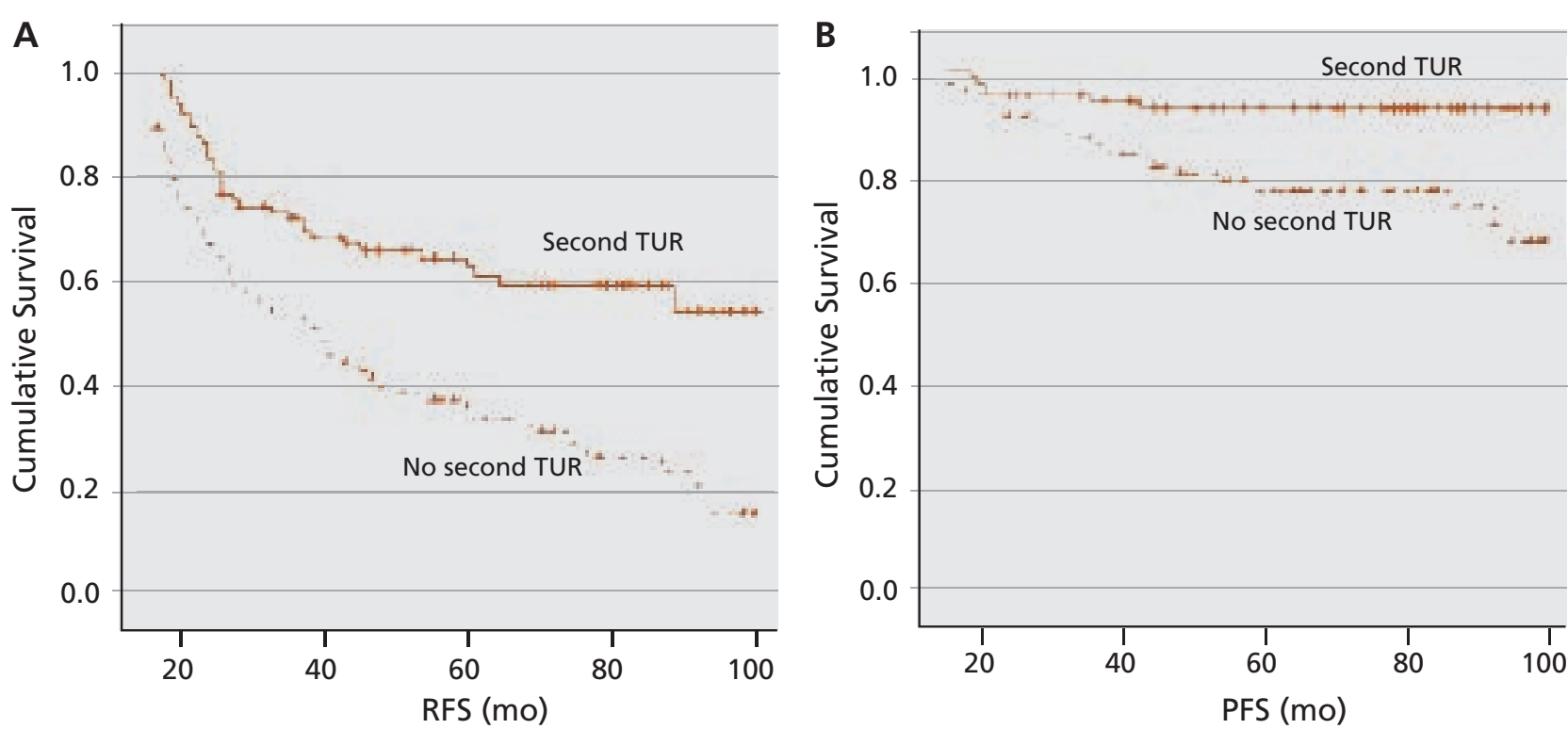

Figure 3 (A) Recurrence-free survival (RFS) and (B) progression-free survival (PFS) in patients with T1 bladder cancer after one versus second transurethral resection (TUR). ${ }^{11}$

fewer recurrences, longer times to tumor recurrence, and less progression than patients who underwent a single TUR. ${ }^{10}$

\section{Repeat TUR is Therapeutic}

Repeat TUR is therapeutic because it facilitates complete resection and improves outcomes. Figure 3 shows results from a prospective randomized trial involving 210 patients with T1 tumors. The second
TUR group had significantly improved recurrencefree survival (52\% vs $21 \%$ ) and progression-free survival (93\% vs $76 \%$ ) compared with the no second TUR group. Although overall survival in both groups was similar, only $2 \%$ of patients died of urothelial cancer after undergoing repeat TUR compared with $11 \%$ after 1 TUR. ${ }^{11}$

Repeat TUR also appears to improve the shortterm response to $\mathrm{BCG}$ therapy. ${ }^{12}$ In a prospective
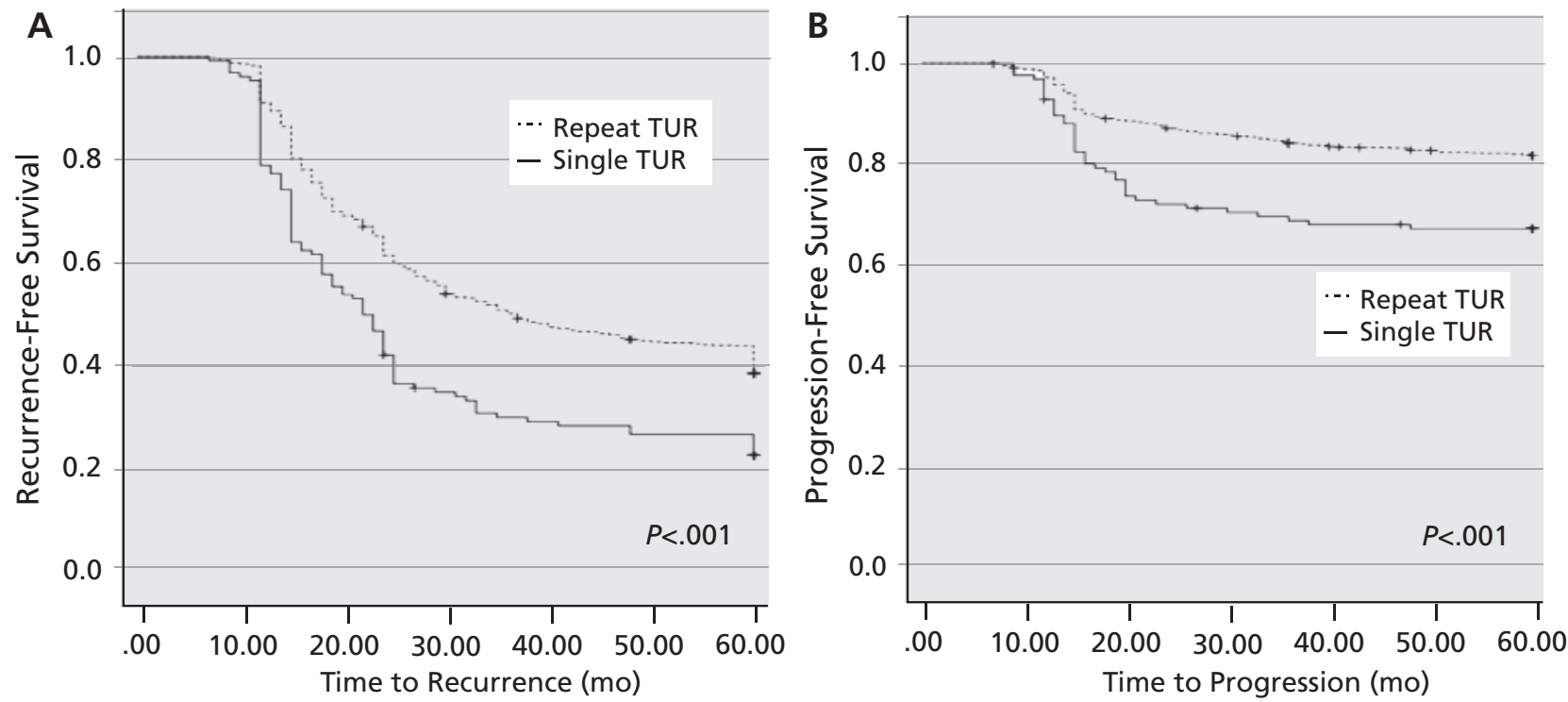

Figure 4 (A) Recurrence-free survival and (B) progression-free survival of patients with non-muscle-invasive bladder cancer after single versus repeat transurethral resection (TUR) and intravesical bacillus Calmette-Guérin therapy. 

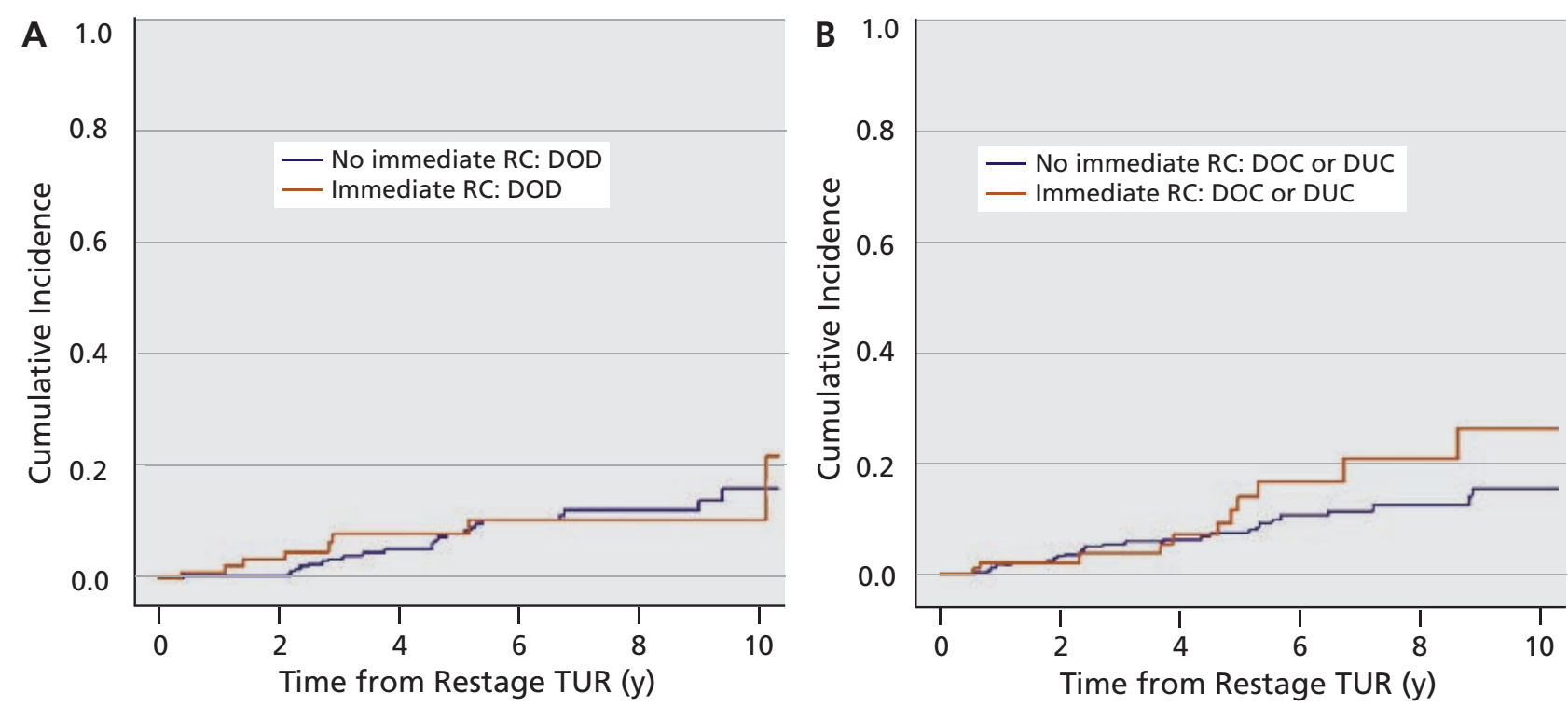

Figure 5 Bladder cancer-specific death in 417 patients with T1 disease stratified by (A) immediate cystectomy versus (B) surveillance or delayed cystectomy.

Abbreviations: DOC, death from other causes; DOD, dead of disease; DUC, death from unknown causes; RC, radical cystectomy; TUR, transurethral resection.

nonrandomized phase II study, $43 \%$ of 127 patients after initial TUR had persistent tumor at 3 months compared with $9.6 \%$ of 894 patients after repeat TUR; by 12 months, $59 \%$ of patients experienced recurrence after 1 TUR compared with $16 \%$ after 2 TURs. Figure 4 shows that overall recurrence-free and progression-free survival was significantly longer with BCG therapy after repeat TUR.

\section{Repeat TUR is Predictive}

Repeat TUR is predictive, because owing to better staging and local tumor control, it leads to changes in therapy that improves outcomes. Figure 5 shows survival of 417 patients with true T1 disease who had repeat TUR and underwent immediate cystectomy or local therapy and surveillance. ${ }^{13}$ Overall and diseasespecific survivals are similar in both groups because patients with T1 disease on repeat TUR underwent immediate cystectomy, whereas patients with no tumor or less than T1 disease on repeat TUR were treated locally and observed. This suggests that repeat TUR identifies patients with high-risk T1 cancer for immediate cystectomy rather than risk tumor progression and decreased survival while pursuing conservative therapy. ${ }^{14}$ Conversely, patients with completely resected or non-T1 disease on repeat TUR can be treated safely by bladder-sparing strategies.

Lastly, patients who have muscle-invasive tumor on repeat TUR require aggressive therapy, such as neoadjuvant chemotherapy and cystectomy. Failure to detect locally invasive bladder cancer may have devastating consequences for individual patients, justifying repeat TUR for all HG tumors.

\section{Conclusions}

Repeat TUR is recommended for any HG NMIBC detected at the initial TUR. Repeat TUR achieves better local control through removing residual disease, provides accurate staging and prognostic information, facilitates response to intravesical therapy, and leads to changes in treatment with better outcomes. A second TUR helps select patients for intravesical bladder-sparing therapy versus cystectomy. Repeat TUR is a diagnostic, therapeutic, prognostic, and predictive procedure, and is essential to successful management of high-risk NMIBC.

\section{References}

1. Herr HW, Donat SM. Quality control in transurethral resection of bladder tumors. BJUI 2008;102:1242-1246.

2. Clark PE, Speiss PE, Agarwal N, et al. NCCN Clinical Practice Guidelines in Oncology: Bladder Cancer. Version 2, 2015. Available at: NCCN.org. Accessed July 15, 2015.

3. Maruniak NA, Takezawa K, Murphy WM. Accurate pathologic staging of urothelial neoplasms requires better cystoscopic sampling. J Urol 2002;167:2404-2407.

4. Zurkirchen MA, Sulser T, Gaspert A, Hauri D. Second TUR of superficial transitional cell carcinoma of the bladder: a must even for experienced urologists. Urol Int 2004;72:99-102. 
Herr

5. Dalbagni G, Herr HW, Reuter VE. Impact of a second transurethral resection on staging of T1 bladder cancer. Urology 2002;60:822-825.

6. Pycha Al. How to teach the teacher to teach the TUR-B. Int J Surg 2007;5:81-85.

7. Richards KA, Smith ND, Steinberg GD. The importance of transurethral resection of bladder tumor in the management of nonmuscle invasive bladder cancer: a systematic review of novel technologies. J Urol 2014;191:1655-1664.

8. Herr HW. Role of re-resection in non-muscle-invasive bladder cancer. ScientificWorldJournal 2011;11:283-288.

9. Vianello A, Costantini E, Del Zingaro M, et al. Repeated white light transurethral resection of the bladder in nonmuscle-invasive urothelial bladder cancers: systematic review and meta-analysis. J Endourol 2011;25:1703-1712.

10. Guevara A, Salomon L, Allory Y, et al. The role of tumor-free status in repeat resection before intravesical BCG for high grade Ta,T1 and CIS bladder cancer. J Urol 2010;183:2161-2164.
11. Divrik RT, Sahin AF, Yildirim U, et al. Impact of routine second TUR on the long-term outcome of patients with newly diagnosed pT1 urothelial carcinoma with respect to recurrence, progression rate, and disease-specific survival: a prospective randomized clinical trial. Eur Urol 2010;58:185190.

12. Sfakianos JP, Kim PH, Herr HW. The effect of restaging transurethral resection on recurrence and progression rates in patients with nonmuscle invasive bladder cancer treated with intravesical BCG. J Urol 2013;191:341-345.

13. Dalbagni G, Vora K, Kaag M, et al. Clinical outcome in a contemporary series of restaged patients with T1 bladder cancer. Eur Urol 2009;56:903910.

14. Herr HW, Donat SM, Dalbagni G. Can restaging transurethral resection of T1 bladder cancer select patients for immediate cystectomy? J Urol 2007;177:75-79. 